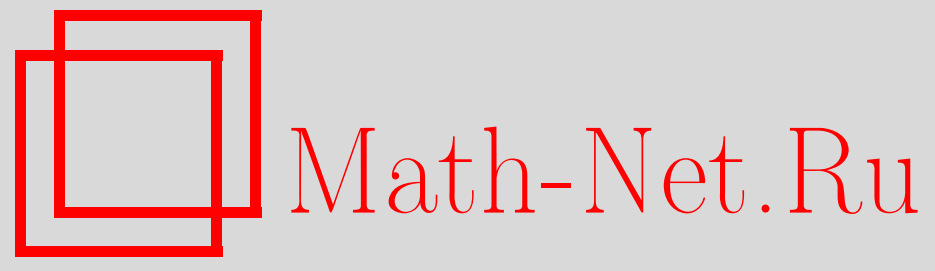

М. В. Давидович, Скорость переноса энергии плоской монохроматической электромагнитной волной через слой вещества, Вестн. Сам. гос. техн. унта. Сер. Физ.-мат. науки, 2020, номер 1, 22-40

DOI: https://doi.org/10.14498/vsgtu1741

Использование Общероссийского математического портала MathNet.Ru подразумевает, что вы прочитали и согласны с пользовательским соглашением

http://www.mathnet.ru/rus/agreement

Параметры загрузки:

IP : 54.174 .149 .18

26 апреля 2023 г., 13:21:08

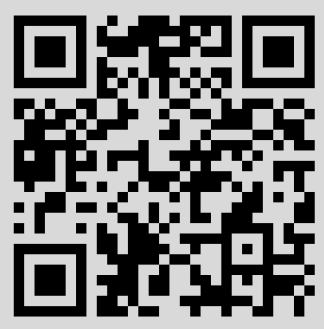


УДК 517.958:530.145

\title{
Скорость переноса энергии плоской монохроматической электромагнитной волной через слой вещества
}

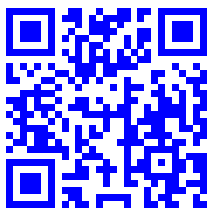

\author{
М. В. Давидович \\ Саратовский государственный университет им. Н. Г. Чернышевского \\ (национальный исследовательский университет), \\ Россия, 410012, Саратов, ул. Астраханская, 83.
}

\begin{abstract}
Аннотация
Рассмотрены стационарные задачи для прохождения (туннелирования) плоской электромагнитной волны через слой вещества с диэлектрическими свойствами, а также и квантовой частицы через прямоугольный потенциальный барьер. Показано, что сверхсветовых движений не возникает, а время прохождения всегда больше времени при прохождении структуры со скоростью света.
\end{abstract}

Ключевые слова: скорость движения энергии, время туннелирования, частотная дисперсия, время Бома-Вигнера, парадокс Хартмана.

Получение: 22 августа 2019 г. / Исправление: 19 ноября 2019 г.

Принятие: 10 февраля 2020 г. / Публикация онлайн: 1 апреля 2020 г.

\section{Введение}

Согласно СТО, скорость движения энергии всегда ограничена скоростью света $c$. Хорошо известно, что перенос энергии плоской монохроматической электромагнитной волной (ЭМВ) в вакууме $\mathbf{E}(z, t)=\mathbf{x}_{0} E_{x} \exp (i \omega(t-z / c))$, $\mathbf{H}=\varepsilon_{0} c \mathbf{z}_{0} \times \mathbf{E}(z, t)$ идет со скоростью света $v_{\mathrm{E}}=S_{z} / W=c$, поскольку усредненные за период плотность электромагнитной энергии $W$ и $z$-компонента вектора Пойнтинга $S_{z}=\operatorname{Re}\left(E_{x} H_{y}^{*}\right) / 2$ выражаются соответственно как $W=$ $=\varepsilon_{0}\left|E_{x}\right|^{2} / 2$ и $S_{z}=\varepsilon_{0} c\left|E_{x}\right|^{2} / 2$. Интересно отметить, что для такой волны в вакууме нет сдвига фаз между действительными полями $\operatorname{Re}(\mathbf{E})$ и $\operatorname{Re}(\mathbf{H})$, поэтому это единственный случай, когда скорость переноса $c$ получается и для мгновенных значений без усреднения. Любая обладающая массой материальная частица имеет внутреннюю (собственную) энергию $m c^{2}$, поэтому движется всегда со скоростью $v<c$. Если имеется поток частиц с постоянной

\section{Научная статья}

(2) (7) Контент публикуется на условиях лицензии Creative Commons Attribution 4.0 International (https://creativecommons.org/licenses/by/4.0/deed.ru)

\section{Образец для цитирования}

Дав и до в и ч М. В. Скорость переноса энергии плоской монохроматической электромагнитной волной через слой вещества // Вестн. Сам. гос. техн. ун-та. Сер. Физ.-мат. науки, 2020. T. 24, № 1. С. 22-40. doi: 10.14498/vsgtu1741.

\section{Сведения об авторе}

Михаил Владимирович Давидович (10) https://orcid.org/0000-0001-8706-8523 доктор физико-математических наук, профессор; профессор; каф. радиотехники и электродинамики; e-mail: DavidovichMV@info.sgu.ru 
плотностью, то эта скорость совпадает со скоростью переноса энергии частицами потока. Соответственно, прохождение частицей в потоке некоторой области $d$ за «сверхсветовое время» $\tau<d / c$ означает сверхсветовой перенос энергии и противоречит СТО. Однако имеется большое количество публикаций в солидных журналах, где приводится утверждение о сверхсветовом туннелировании ЭМВ, а также и о сверхсветовом туннелировании частиц (см., например, [1-36] и ссылки на литературу там), в том числе о мгновенном туннелировании, и даже об отрицательном времени задержки (запаздывания) [37-42]. Отрицательная задержка (т. е. опережение следствием его причины) противоречит принципу причинности даже в предположении дальнодействия или бесконечной скорости распространения [43]. В подтверждение указанных утверждений приводятся эксперименты [20-23,30-36], поэтому теоретическое рассмотрение задач туннелирования весьма важно, как и объяснение (интерпретация) указанных экспериментов. Любой эксперимент всегда длится конечное время и связан с нестационарной постановкой, т. е. и с нестационарной задачей. Строгое экспериментальное измерение стационарной скорости $c=S_{z} / W$ невозможно, поскольку требует прерывания волны, а это уже нестационарный случай. Прерывание волны образует цуги, или волновые пакеты (ВП), и результат будет тем точнее, чем длиннее цуг. Поэтому точное подтверждение факта $v \leqslant c$ в монохроматической волне возможно только теоретически, благо для ЭМВ есть строгий и точный инструмент уравнения Максвелла. Рассмотрение нестационарного квантового туннелирование как частиц с массой, так и фотонов усложняется тем, что имеет место нелокальность волновой функции и ВП [27]. Вопросы нестационарного туннелирования требуют отдельного рассмотрения и не исследуются в данной работе. Целью работы является определение скорости переноса энергии монохроматической ЭМВ через слой вещества и получение для нее условия $v_{\mathrm{E}} \leqslant c$.

В работе в строгой постановке на основе уравнений Максвелла с учетом материальной дисперсии рассмотрена и решена простая задача о дифракции падающей из вакуума плоской монохроматической ЭМВ на однородном слое вещества в области $0 \leqslant z \leqslant d$ с заданной диэлектрической проницаемостью (ДП) $\varepsilon(\omega)$, на основе чего получена скорость переноса энергии такой волной через слой, которая меньше скорости света. Этот результат получен и для туннелирования через слой холодной столкновительной плазмы. При постановке задачи анизотропными, бианизотропными и магнитными свойствами слоя мы пренебрегаем, пространственную дисперсию не рассматриваем.

\section{Постановка и решение задачи}

При падении из вакуума ЭМВ с координатной зависимостью $\exp \left(-i k_{x} x-\right.$ $\left.-i k_{0 z} z\right)$ под углом $\theta=\arctan \left(k_{x} / k_{0 z}\right)$ на слой имеют место соотношения $k_{0 z}=\sqrt{k_{0}^{2}-k_{x}^{2}}, E_{0 z}=|E| \sin (\theta), E_{x}= \pm|E| \cos (\theta)$, где $|E|=\sqrt{E_{x}^{2}+E_{y}^{2}}-$ амплитуда ЭМВ. Знак «минус» соответствует отраженной волне. В слое величина $k_{z}=\sqrt{k_{0}^{2} \varepsilon-k_{x}^{2}}$ может быть комплексной, поэтому для прошедшей волны $\theta^{\prime}=\arctan \left(k_{x} / k_{z}\right), E_{x} / E_{z}=k_{z} / k_{x},|E|=\left|E_{x}\right| \sqrt{1+\left|k_{z}\right|^{2} / k_{x}^{2}}$. Вводим, как обычно, нормированные волновые сопротивления $\rho_{0}^{e}=k_{0 z} / k_{0}, \rho^{e}=k_{z} /\left(k_{0} \varepsilon\right)$ для Е-мод, и $\rho_{0}^{h}=k_{0} / k_{0 z}, \rho^{h}=k_{0} / k_{z}$ для Н-мод соответственно в вакууме и в пластине. Первый случай соответствует $p$-поляризации $\rho^{e}=E_{x} /\left(Z_{0} H_{y}\right)$, 
а второй $-s$-поляризации $\rho^{h}=-E_{y} /\left(Z_{0} H_{x}\right)$. Здесь $Z_{0}=\sqrt{\mu_{0} / \varepsilon_{0}}=1 /\left(\varepsilon_{0} c\right)$.

Далее будем использовать нормировку $\tilde{\rho}=\rho / \rho_{0}$. При нормальном падении $k_{x}=0, \rho^{e}=\rho^{h}$ и $\tilde{\rho}^{(e, h)}=\rho^{(e, h)}$. При падении из вакуума всегда $k_{x} \leqslant k_{0}=\omega / c$.

Для задач туннелирования ЭМВ при нарушенном полном внутреннем отражении $[28,29]$ интересен случай падении волны из диэлектрика на вакуумный зазор. Тогда $k_{0} \leqslant k_{x} \leqslant k_{0} \varepsilon$, величина $k_{z}=\sqrt{k_{0}^{2} \varepsilon-k_{x}^{2}}$ - действительная, a $k_{0 z}=\sqrt{k_{0}^{2}-k_{x}^{2}}=-i \sqrt{k_{x}^{2}-k_{0}^{2}}-$ мнимая. Решение стационарной (монохроматической) задачи для однородного слоя весьма простое - коэффициент прохождения дается формулой

$$
T(\omega)=|T| \exp (j \phi)=\left[\cos (k d)+i \sin (k d)\left(\tilde{\rho}+\tilde{\rho}^{-1}\right) / 2\right]^{-1},
$$

а коэффициент отражения имеет вид

$$
R(\omega)=(Z-1) /(Z+1), \quad Z=\tilde{\rho} \alpha^{+} / \alpha^{-}, \quad \alpha^{ \pm}=(1+\tilde{\rho}) \pm \exp (-2 i k d)(1-\tilde{\rho}) .
$$

При этом внутри слоя $E(z)=A^{+} \exp (-i k z)+A^{-} \exp (i k z)$, где амплитуды имеют вид $A^{ \pm}=|T|(1 \pm \tilde{\rho}) \exp ( \pm i k d) / 2$.

Мы рассмотрим простой закон дисперсии Лоренца для однородного слоя

$$
\varepsilon(\omega)=1+\frac{\omega_{p}^{2}}{\omega_{0}^{2}-\omega^{2}+i \omega \omega_{c}} .
$$

Для него обратное преобразование Фурье определяет ядро $\varepsilon(t)$ интегрального оператора, связывающего электрическое поле и индукцию во времени [44]:

$$
\varepsilon(t)=\delta(t)+\chi(t) \frac{\omega_{p}^{2} \exp \left(-\omega_{c} t / 2\right)}{\sqrt{\omega_{0}^{2}-\omega_{c}^{2} / 4}} \sin \left(t \sqrt{\omega_{0}^{2}-\omega_{c}^{2} / 4}\right) .
$$

Для реальных сред дисперсия ДП содержит несколько таких членов с учетом локального поля и нескольких частот $\omega_{0}, \omega_{p}, \omega_{c}$. Величины $\omega_{p}^{2}$ определяются через силы осцилляторов соответствующих квантовых переходов с частотами $\omega_{0}$, а величины $\omega_{c}$ и $\tau_{c}=1 / \omega_{c}$ соответствуют ширинам соответствующих спектральных линий и временам жизни возбужденных состояний. Полагая $\omega_{p}=0$ (несвязанные заряды диполя), получаем $\varepsilon(t)=\delta(t)+$ $+\omega_{p}^{2} \omega_{c}^{-1}\left[1-\exp \left(-\omega_{c} t\right)\right]$, что совпадает с обратным преобразованием Фурье от дисперсии ДП $\varepsilon(\omega)=\varepsilon_{L}-\omega_{p}^{2} /\left(\omega^{2}-j \omega \omega_{c}\right)$ Друде для плазмы. Для газовой плазмы $\varepsilon_{L}=1$. Для плазмы в металле вклад в $\varepsilon_{L} \sim 10$ дают другие члены дисперсии Лоренца, взятые на низких частотах. В общем случае терм $\varepsilon_{L}$ определяет вклад переходов уровней в валентной зоне и межзонных переходов. При $\omega<\omega_{p} / \sqrt{\varepsilon_{L}}$ и малой частоте столкновений $\omega_{c} \ll \omega$ ДП плазмы становится почти отрицательной, и возможно стационарное туннелирование ЭМВ. Для дисперсии Лоренца условие $\varepsilon^{\prime}(\omega)=\operatorname{Re}(\varepsilon(\omega))<0$, при котором в соответствующем диапазоне имеет место стационарное туннелирование, также возможно. Нормируя все частоты на плазменную частоту и обозначая их большой буквой омега, видим, что оно при предельно малой частоте столкновений выполнено, если $\Omega_{0}<\Omega<\sqrt{1+\Omega_{0}^{2}}$, а при конечном времени жизни это область нормированных частот $\left|\Omega^{2}-\Omega_{0}^{2}-\left(1-\Omega_{c}^{2}\right) / 2\right| \leqslant \sqrt{\left(1-\Omega_{c}^{2}\right)^{2} / 4-\Omega_{0}^{2} \Omega_{c}^{2}}$. 
В случае большой диссипации $\Omega_{c}>\left|1-\Omega_{c}^{2}\right| /\left(2 \Omega_{0}\right)$ (малой силы осциллятора) область отрицательных значений ДП для дисперсии Лоренца отсутствует.

Рассматриваем туннелирование для слоя из газовой плазмы или плазмы металлов и полупроводников. Для последних в определении плазменной частоты следует использовать эффективные массы электронов и дырок. Туннелированию соответствует условие $\varepsilon^{\prime}(\omega)<0$ в ДП $\varepsilon(\omega)=\varepsilon^{\prime}(\omega)-i \varepsilon^{\prime \prime}(\omega)$, $\varepsilon^{\prime \prime}(\omega)>0$, при этом в простейшем случае нормального падения $\rho=1 / \sqrt{\varepsilon}$, $k_{z}=k_{0} \sqrt{\varepsilon(\omega)}=k_{z}^{\prime}-i k_{z}^{\prime \prime}$, где

$$
k_{z}^{\prime}=\frac{\sqrt{2}}{k_{0}} \sqrt{\sqrt{\varepsilon^{\prime 2}+\varepsilon^{\prime \prime 2}}+\varepsilon^{\prime}}, \quad k_{z}^{\prime \prime}=\frac{\sqrt{2}}{k_{0}} \sqrt{\sqrt{\varepsilon^{\prime 2}+\varepsilon^{\prime \prime 2}}-\varepsilon^{\prime}} .
$$

При туннелировании всегда $k_{z}^{\prime \prime}>k_{z}^{\prime}$, а в приближении отсутствия диссипации $k_{z}^{\prime}=0$, т. е. прямая волна экспоненциально затухающая (эванесцентная), а обратная - экспоненциально нарастающая (антиэванесцентная). Ее амплитуда с ростом толщины $d$ экспоненциально падает, но наличие этой волны и ее интерференция с прямой и приводят к туннелированию, т. е. к просачиванию энергии через слой.

Для случая падения под углом $k_{z}=k_{0} \sqrt{\varepsilon-\left(k_{x} / k_{0}\right)^{2}}$ формулы (2) следует модифицировать путем замены $\varepsilon^{\prime} \rightarrow \varepsilon^{\prime}-\left(k_{x} / k_{0}\right)^{2}$. При туннелировании удобно сделать замену $k_{z}=-i k_{0} \sqrt{\left(k_{x} / k_{0}\right)^{2}-\varepsilon}$. Тогда при условии $\varepsilon^{\prime}<0$ и при пренебрежении диссипацией имеем $\rho=i / \sqrt{|\varepsilon|}, k_{z}=-i k_{0} \sqrt{\left(k_{x} / k_{0}\right)^{2}+|\varepsilon|}$. В общем случае $k_{z}=k_{z}^{\prime}-i k_{z}^{\prime \prime}, \tilde{\rho}=\tilde{\rho}^{\prime}+i \tilde{\rho}^{\prime \prime}, k_{z}^{\prime \prime}>0, \tilde{\rho}^{\prime}>0$, а амплитуды волн обоих направлений в пластине выражаются в виде $A^{ \pm}=\exp ( \pm i k d) T(1 \mp \tilde{\rho}) / 2$.

Для плоской волны в среде также вводим скорость движения энергии по формуле $v_{\mathrm{E}}=S_{z} / W$. Здесь также $S_{z}=\operatorname{Re}\left(E_{x} H_{y}^{*}\right) / 2$, если энергия переносится вдоль оси $z$ и выбрана поляризация $\mathbf{E}=\mathbf{x}_{0} E_{x}$. Заметим, что при движении под углом $v_{\mathbf{E} z}=v_{\mathbf{E}} \cos (\theta)$. Если в диссипативной среде нет накопленной кинетической энергии колебаний (например, в дистиллированной воде, описываемой формулой Дебая), то $W=\varepsilon_{0}\left(\varepsilon^{\prime}+\sqrt{\varepsilon^{\prime 2}+\varepsilon^{\prime \prime 2}}\right)\left|E_{x}\right|^{2} / 4$ [45], поэтому $v_{\mathrm{E}}=c / \sqrt{\left(\sqrt{\varepsilon^{\prime 2}+\varepsilon^{\prime \prime 2}}+\varepsilon^{\prime}\right) / 2}<c$. Без диссипации $v_{\mathrm{E}}=c / \sqrt{\varepsilon^{\prime}}$. Диссипация уменьшает $|T|$ и увеличивает замедление. Для столкновительной плазмы имеем [45-47]

$$
\begin{gathered}
W=\frac{1}{4} \varepsilon_{0}\left|E_{x}\right|^{2}\left[2-\varepsilon^{\prime}+\sqrt{\varepsilon^{\prime 2}+\varepsilon^{\prime \prime 2}}\right], \\
S_{z}=\frac{1}{\sqrt{8}} c \varepsilon_{0}\left|E_{x}\right|^{2} \sqrt{\sqrt{\varepsilon^{\prime 2}+\varepsilon^{\prime \prime 2}}+\varepsilon^{\prime}}, \\
v_{\mathrm{E}}=\frac{c\left[\left(\sqrt{\varepsilon^{\prime 2}+\varepsilon^{\prime \prime 2}}+\varepsilon^{\prime}\right) / 2\right]^{1 / 2}}{1+\left(\sqrt{\varepsilon^{\prime 2}+\varepsilon^{\prime \prime 2}}-\varepsilon^{\prime}\right) / 2} .
\end{gathered}
$$

Для нее $\varepsilon^{\prime}=1-\omega_{p}^{2} /\left(\omega^{2}+\omega_{c}^{2}\right), \varepsilon^{\prime \prime}=\omega_{p}^{2} \omega_{c} /\left(\omega^{3}+\omega \omega_{c}^{2}\right)$. Поэтому для плазмы без диссипации $W=\varepsilon_{0}\left|E_{x}\right|^{2}\left(1+\omega_{p}^{2} / \omega^{2}\right) / 2$. В (3), (4) следует иметь в виду, что из-за диссипации амплитуда зависит от координаты как $\left|E_{x}(z)\right|=$ $=\left|E_{x}(0)\right| \exp \left(-k^{\prime \prime} z\right)$. Увеличение электрической энергии такой плазмы по сравнению с энергией в вакууме связано с кинетической энергией колебаний ее частиц с частотой $\omega[46]$. На плазменной частоте электрическая часть 
энергии в два раза больше, чем в вакууме, а магнитная часть равна нулю. При пренебрежении диссипацией ниже плазменной частоты $v_{\mathrm{E}}=0$, a выше нее $v_{\mathrm{E}}=c \sqrt{1-\omega_{p}^{2} / \omega^{2}}$, что совпадает с групповой скоростью (ГС). Ниже плазменной частоты $\Gamma$ С мнимая, а $v_{\mathrm{E}}=0$, т. е. распространение невозможно. При малой диссипации разлагаем (3) и (4) по малому параметру $\varepsilon^{\prime \prime 2}$, считая $\left|\varepsilon^{\prime}\right| \geqslant 1$. Тогда получаем

$$
S_{z}=c \varepsilon_{0}\left|E_{x}\right|^{2} \varepsilon^{\prime \prime} /\left(4\left|\varepsilon^{\prime}\right|\right), \quad W=\varepsilon_{0}\left|E_{x}\right|^{2}\left[1+\left|\varepsilon^{\prime}\right|+\varepsilon^{\prime \prime 2} /\left(4\left|\varepsilon^{\prime}\right|\right)\right] / 2,
$$

и в такой слабодиссипативной плазме $v_{\mathrm{E}}=c \varepsilon^{\prime \prime} /\left(2\left|\varepsilon^{\prime}\right|+2\left|\varepsilon^{\prime}\right|^{2}+\varepsilon^{\prime \prime 2} / 2\right)$. Заметим, что слабая диссипация возможна только в области $\omega \gg \omega_{c}$. Вблизи плазменной частоты $\varepsilon^{\prime} \approx 0$, применять разложение нельзя, и непосредственно из (3) и (4) имеем $v_{\boldsymbol{E}}=c \sqrt{\varepsilon^{\prime \prime} / 2} /\left(1+\varepsilon^{\prime \prime} / 2\right) \ll c$. Средой без накопления энергии колебаний можно считать и плазму на низких частотах $\omega \ll \omega_{c}$ [48]. Тогда $\varepsilon(\omega)=-i \omega_{p}^{2} /\left(\omega \omega_{c}\right)=-i \sigma \varepsilon_{0} / \omega$, и из $(5)$ следует $v_{\mathrm{E}} \approx 2 c \sqrt{\omega \omega_{c}} / \omega_{p} \ll c$. Дисперсия в такой среде обусловлена проводимостью $\sigma$. Можно получить аналогичное (5) выражение для $v_{\mathrm{E}}$ в случае дисперсии Лоренца, для которой также всегда $v_{\mathrm{E}}<c$. Соответствующее громоздкое выражение мы не приводим.

В области аномальной отрицательной дисперсии при малой диссипации для такой среды также возможен случай $\varepsilon^{\prime}<0$ и туннелирование. Отметим, что из дисперсии Лоренца при равной нулю резонансной частоте $\omega_{0}=0$ (свободные осцилляторы) следует дисперсия плазмы, а предельный переход $\omega_{0} \rightarrow \infty, \omega_{c} \rightarrow \infty, \omega_{p} \rightarrow \infty$ (бесконечно жесткие диполи) при условии $\omega_{p}^{2} / \omega_{0}^{2}=\kappa, \omega_{c}^{2} / \omega_{0}^{4}=\tau^{2}$ дает формулу Дебая $\varepsilon^{\prime}=1+\kappa /\left(1+\omega^{2} \tau^{2}\right)$, $\varepsilon^{\prime \prime}=\left(\varepsilon^{\prime}-1\right) \omega \tau[45]$. Отметим также, что при движении под углом скорость переноса энергии вдоль $z$ всегда меньше, чем в направлении движения. Примером служат волны в волноводах, которые состоят из двигающихся под углом и отражающихся от стенок плоских волн.

Покажем, что туннелирование сквозь слой бесстолкновительной плазмы идет со скоростью меньше скорости света как ниже плазменной частоты, так и выше нее, при этом плотность потока мощности пропорциональна $|T|^{2}$ и непрерывна. При диссипации эта скорость уменьшается и более существенно зависит от координаты. ГС соответствует скорости распространения энергии только в монохроматической волне и только в абсолютно недиссипативных (консервативных или гамильтоновых) однородных системах и средах [5]. Только в этих случаях ГС есть величина действительная и преобразующаяся как полярный вектор, т. е. как скорость материальной точки. В полосах непрозрачности, в том числе и внутри барьеров, ГС есть величина кинематическая, определяющая скорость движения биений двух бесконечно близких по частоте волн и может быть любой - превышающей $c$, бесконечной и даже отрицательной (направленной против движения энергии) [50]. Это же относится и к групповому времени запаздывания, которое может стать нулевым и отрицательным [43]. Движение же ВП и скорость переноса пакетом энергии, особенно при достаточно широком спектре, не следует отождествлять с ГС. Очевидно, энергетическая скорость $v_{\mathrm{E}}(z)=S_{z}(z) / W(z)$ в неоднородной среде и при наличии границ раздела не является постоянной величиной. Наиболее простой вид она имеет для прозрачного диэлектрического слоя без 
дисперсии с ДП $\varepsilon=$ const $>1$ :

$$
v_{\mathrm{E}}(z)=2 c /\left[\varepsilon+1+(\varepsilon-1) \cos \left(2 k_{0} \sqrt{\varepsilon}(z-d)\right)\right] \leqslant c .
$$

На границах слоя имеем

$$
v_{\mathrm{E}}(d)=c / \varepsilon<c / \sqrt{\varepsilon}, \quad v_{\mathrm{E}}(0)=2 c /\left[\varepsilon+1+(\varepsilon-1) \cos \left(2 k_{0} \sqrt{\varepsilon} d\right)\right] .
$$

При длине $d=m \lambda /(2 \sqrt{\varepsilon})$, т. е. кратной целому числу полуволн в среде, получаем $v_{\mathrm{E}}(0)=c / \varepsilon$. В этом случае при $m=1$ имеем так называемую полуволновую «банку», известную из электроники СВЧ как полностью прозрачное устройство - для нее $T=1$.

При длине $d=(2 m-1) \lambda /(4 \sqrt{\varepsilon})$, т. е. кратной нечетному числу четвертьволновых длин в среде, имеем $v_{\mathrm{E}}(0)=c$. В точках, где косинус равен единице, имеем $v_{\mathrm{E}}(z)=c / \varepsilon$, в точках, где он равен минус единице, будет $v_{\mathrm{E}}(z)=c$, а в точках, где он равен нулю, получаем $v_{\mathrm{E}}(z)=2 c /(\varepsilon+1)<c / \sqrt{\varepsilon}$. При $\varepsilon=1$ везде $v_{\mathrm{E}}(z)=c$.

В среде энергию переносят квазифотоны или поляритоны. Найдем усредненную скорость квазифотона через слой:

$$
\bar{v}_{\mathrm{E}}=d^{-1} \int_{0}^{d} v_{\mathrm{E}}(z) d z=\frac{c}{k_{0} \sqrt{\varepsilon} d} \int_{0}^{2 k_{0} d \sqrt{\varepsilon}} \frac{d x}{\varepsilon+1+(\varepsilon-1) \cos (x)} .
$$

При оптической толщине слоя $\theta=k_{0} d \sqrt{\varepsilon} \leqslant \pi / 2$ интеграл (6) вычисляется (см. [51], формула 1.5.9.15) и имеет вид $\bar{v}_{\mathrm{E}}=c\left(k_{0} d \varepsilon\right)^{-1} \arctan \left(\varepsilon^{-1 / 2} \tan \left(k_{0} d \sqrt{\varepsilon}\right)\right)$. При малой оптической толщине $\theta \ll 1$ получаем $\bar{v}_{\mathrm{E}}=c / \varepsilon$, а при большой оптической толщине пишем $\theta=m \pi / 2+\Delta \theta, d=(m \pi / 2+\Delta \theta) /\left(k_{0} \sqrt{\varepsilon}\right)$, интеграл (6) разбиваем на $m$ участков длины $2 \theta$ плюс интеграл по $2 \Delta \theta$, и в пределе $m \rightarrow \infty$ получаем для толстого слоя также $\bar{v}_{\mathrm{E}}=c / \varepsilon$. Если рассмотреть «монохроматическую» волну $E(z, t)=\theta(\omega(t-z / c)) \sin (\omega(t-z / c))$ с резким фронтом $(\theta$ - функция Хэвисайда), то скорость прохождения ее фронта в слое $v=c / \sqrt{\varepsilon}$. Бесконечно тонкая граница раздела не вносит задержку. Время прохождения фронта $\tau=d / v=d \sqrt{\varepsilon} / c$ и следует отождествлять с искомым временем. Реальная волна содержит высокие частоты, а в реальной среде есть дисперсия. Поэтому высокочастотные фотоны предвестника проходят слой за время $\tau_{c}=d / c$. Отражение от второй границы снижает среднюю скорость. Многократные отражения приводят к полученному результату. Время туннелирования для него определим как

$$
\tau_{\mathrm{E}}=\int_{0}^{d} \frac{d z}{v_{\mathrm{E}}(z)}=\frac{d}{c} \frac{1+\varepsilon+(\varepsilon-1) \operatorname{sinc}\left(2 k_{0} d \sqrt{\varepsilon}\right)}{2} .
$$

При предельно малой оптической толщине $\tau_{\mathrm{E}}=\varepsilon d / c$. При малой оптической толщине с учетом двух членов разложения в синусе $\tau_{\mathrm{E}}=(d / c) \times$ $\times\left[\varepsilon-\left(k_{0} d\right)^{2} \varepsilon / 3\right]$. При большой оптической толщине $\tau_{\mathrm{E}}=(d / c)(\varepsilon+1) / 2>$ $>d /(c / \sqrt{\varepsilon})$, т. е. прохождение через слой занимает больше времени, чем движение волны на эквивалентном участке в вакууме. Отметим, что в полученные соотношения нельзя подставлять отрицательные ДП и получать комплексные времена. 
В случае туннелирования через недиссипативный слой с отрицательной ДП пишем

$$
\begin{gathered}
E_{x}=A^{+} \exp \left(-k^{\prime \prime} z\right)+A^{-} \exp \left(k^{\prime \prime} z\right), H_{y}=-i c \varepsilon_{0} \sqrt{|\varepsilon|}\left(A^{+} \exp \left(-k^{\prime \prime} z\right)-A^{-} \exp \left(k^{\prime \prime} z\right)\right), \\
k^{\prime \prime}=k_{0} \sqrt{|\varepsilon|}, \quad A^{ \pm}=\exp \left( \pm k^{\prime \prime} d\right) T(1 \pm i / \sqrt{|\varepsilon|}) / 2
\end{gathered}
$$

поэтому

$$
\begin{gathered}
S_{z}(z)=c \varepsilon_{0}|T|^{2} / 2, \quad W=\varepsilon_{0}|E|^{2}(1+|\varepsilon|) / 2 \\
|E|^{2}=\left[(|\varepsilon|+1) \cosh \left(2 k^{\prime \prime}(z-d)\right)+|\varepsilon|-1\right]|T|^{2} /(2 /|\varepsilon|)
\end{gathered}
$$

и для скорости энергии на частоте ниже плазменной

$$
v_{\mathrm{E}}(\omega)=c \frac{\left(\omega_{p}^{2} / \omega^{2}-1\right)}{\left(\omega_{p}^{2} / \omega^{2}\right)\left[\omega_{p}^{2} / \omega^{2} \cosh ^{2}\left(k^{\prime \prime}(z-d)\right)-1\right]} .
$$

При $z=d$ имеем $v_{\mathrm{E}}(\omega)=c \omega^{2} / \omega_{p}^{2}<c$. Имеем также $v_{\mathrm{E}}\left(\omega_{p}\right)=0$, а при $\omega \ll \omega_{p}$ получаем $v_{\mathrm{E}}(\omega) / c \approx \omega^{2} /\left[\omega_{p}^{2} \cosh ^{2}\left(k^{\prime \prime}(z-d)\right)-\omega^{2}\right]$. Эта скорость очень мала, особенно в начале широкого барьера, где она мала экспоненциально.

Рассмотрим время туннелирования

$$
\tau_{\mathrm{E}}=\int_{0}^{d} \frac{d z}{v_{\mathrm{E}}(\omega)}=\frac{\left(\omega_{p}^{2} / \omega^{2}\right)}{\omega\left(\omega_{p}^{2} / \omega^{2}-1\right)^{2}}\left[\omega_{p}^{2} / \omega^{2} \sinh \left(2 k^{\prime \prime} d\right) / 4-k^{\prime \prime} d / 2\right] .
$$

Для широких барьеров $\tau_{\mathrm{E}}=\left(\omega_{p}^{4} / \omega^{5}\right) \exp \left(2 k_{0} d\left(\omega_{p}^{2} / \omega^{2}-1\right)\right) /\left[2\left(\omega_{p}^{2} / \omega^{2}-1\right)^{2}\right]$, т. е. это время экспоненциально большое. Для узкого барьерного слоя $\tau_{\mathrm{E}}=$ $=(d / c)\left(\omega_{p}^{2} / \omega^{2}\right) / \sqrt{4\left(\omega_{p}^{2} / \omega^{2}-1\right)}$. На плазменной частоте оно бесконечное.

Для широких барьеров коэффициент $|T|^{2}$ экспоненциально мал, поэтому $|R|^{2}=1-|T|^{2} \approx 1$. Это означает наличие в вакууме перед барьером двух приблизительно равных и противоположных потоков. Поскольку перед барьером $E=\exp \left(-j k_{0} z\right)+R \exp \left(j k_{0} z\right)$, то $|E|^{2}=1+|R|^{2}+2|R| \cos \left(2 k_{0} z+\varphi\right)$, $S_{z}=c \varepsilon_{0}|T|^{2} / 2$, и $v_{\mathrm{E}}(\omega) / c$ изменяется от $(1-|R|) /(1+|R|)$ до $(1+|R|) /(1-|R|)$. Здесь $R=|R| \exp (j \varphi)$. Очевидно, сверхсветовые значения здесь связаны с отражением от барьера и интерференцией. При отдельном рассмотрении потоков их скорость равна $c$. Усредним эту скорость по области между двумя ближайшими точками $-z_{1}$ и $-z_{2}$, для которых $l=z_{1}-z_{2}=\pi / k_{0}$. Выберем точку $-z_{1}$ так, что в ней косинус обращается в единицу. Тогда (см. [52], формула 3.792.1)

$$
\begin{gathered}
\frac{\pi \bar{v}_{\mathrm{E}}(\omega)}{c k_{0}}=\int_{-z_{1}}^{-z_{1}+\pi / k_{0}} \frac{\left(1-|R|^{2}\right) d z}{1+|R|^{2}+2|R| \cos \left(2 k_{0}\left(z+z_{1}\right)+\pi\right)}= \\
=\int_{-\pi}^{\pi} \frac{\left(2 k_{0}\right)^{-1}\left(1-|R|^{2}\right) d x}{1+|R|^{2}+2|R| \cos (x)}=\frac{\pi}{k_{0}}
\end{gathered}
$$

или $\bar{v}_{\mathrm{E}}(\omega)=c$. В случае диссипативной пластины соотношения усложняются, и мы их не приводим. Но и в этом случае нет сверхсветовых скоростей. Слабая диссипация уменьшает скорость в высокопрозрачном слое. 
Рассмотрим туннелирование через вакуумный зазор при условии НПВО. В этом случае для $p$-поляризации внутри зазора

$$
\begin{gathered}
W=\varepsilon_{0}\left|E_{x}\right|^{2}\left(1+k_{x}^{2} /\left|k_{0 z}\right|^{2}\right) / 4+\mu_{0}\left|H_{y}\right|^{2} / 4, \quad\left|H_{y}\right|^{2}=\left(\varepsilon_{0} / \mu_{0}\right)\left|E_{x}\right|^{2} /\left|\rho_{0}\right|^{2}, \\
\rho_{0}=k_{0 z} / k_{0}, \quad k_{0 z}=\sqrt{k_{0}^{2}-k_{x}^{2}}=-i \sqrt{k_{x}^{2}-k_{0}^{2}} .
\end{gathered}
$$

Очевидно,

$$
\begin{array}{r}
\left|E_{x}\right|^{2}=\frac{|T|^{2}}{4}\left|\left(1-i\left|\rho_{0}\right|\right) \exp \left(-\left|k_{0 z}\right|(z-d)\right)+\left(1+i\left|\rho_{0}\right|\right) \exp \left(\left|k_{0 z}\right|(z-d)\right)\right|^{2}= \\
=\frac{|T|^{2}}{2}\left(\left(1+\left|\rho_{0}\right|^{2}\right) \cosh \left(2\left|k_{0 z}\right|(z-d)\right)+1-\left|\rho_{0}\right|^{2}\right) .
\end{array}
$$

Вычисляем компоненту вектора Пойнтинга:

$$
\begin{array}{r}
E_{x} H_{y}^{*}=\frac{c \varepsilon_{0}|T|^{2}}{4 i\left|\rho_{0}\right|}\left[\left(1-i\left|\rho_{0}\right|\right) \exp \left(-\left|k_{0 z}\right|(z-d)\right)+\left(1+i\left|\rho_{0}\right|\right) \exp \left(\left|k_{0 z}\right|(z-d)\right)\right] \times \\
\times\left[\left(1+i\left|\rho_{0}\right|\right) \exp \left(-\left|k_{0 z}\right|(z-d)\right)-\left(1-i\left|\rho_{0}\right|\right) \exp \left(\left|k_{0 z}\right|(z-d)\right)\right],
\end{array}
$$

имеем $S_{z}=|T|^{2} / 2$. Отсюда получаем

$$
v_{\mathrm{E}}(z)=\frac{2 c}{\left(\left(1+\left|\rho_{0}\right|^{2}\right) \cosh \left(2\left|k_{0 z}\right|(z-d)\right)+1-\left|\rho_{0}\right|^{2}\right)\left(1+k_{x}^{2} /\left(2\left|k_{0 z}\right|^{2}\right)\right)} .
$$

При бесконечно малом зазоре заменяем косинус гиперболический единицей и получаем $v_{\mathrm{E}}(z)=c /\left(1+k_{x}^{2} /\left(2\left|k_{0 z}\right|^{2}\right)\right)<c$ или $v_{\mathrm{E}}(z)=c\left(2 k_{x}^{2}-2 k_{0}^{2}\right) /\left(3 k_{x}^{2}-2 k_{0}^{2}\right)$. Эта же скорость соответствует концу слоя $v_{\mathrm{E}}(d)$. При широком зазоре вдали от конца слоя скорость становится экспоненциально малой, а время туннелирования - экспоненциально большим. Не представляет труда получить аналогичные результаты для $s$-поляризованного туннелирования.

В ряде работ, например [53-56], получены решения для неоднородного слоя с ДП $\varepsilon(z)$. Такой слой можно представить как совокупность однородных слоев, определить скорость энергии в каждом слое и время по формулам типа (7), (8). Для плазмы следует предположить, что плазменная частота зависит от координаты. Поскольку для каждого из слоев скорость «досветовая», то и для всей структуры $v_{\mathrm{E}} \leqslant c$. Это, например, имеет место и для запрещенной зоны фотонного кристалла $(\Phi \mathrm{K})$ с двумя прозрачными слоями в периоде и несколькими десятками периодов. Для такого ФК можно произвести гомогенизацию, и тогда в его запрещенной зоне эффективная ДП $\varepsilon_{\text {eff }}<0$, поэтому задачу приближенно можно свести к задаче для одного слоя. Заметим, что отражения от границ раздела только замедляют передачу энергии. Для решения удобен метод матриц передачи [53]. Указанную задачу можно также решить и методом интегрального уравнения (ИУ) вида

$$
E_{x}(z)=E_{0} \exp \left(-i k_{0} z\right)+\int_{0}^{d} G_{\omega}\left(z-z^{\prime}\right)\left(\varepsilon\left(z^{\prime}\right)-1\right) E_{x}\left(z^{\prime}\right) d z^{\prime} .
$$

В нем обозначено ядро $G_{\omega}(z)=-i k_{0} \exp \left(-i k_{0}|z|\right) / 2$, удовлетворяющее уравнению $\left(\partial_{z}^{2}+k_{0}^{2}\right) G_{\omega}(z)=-k_{0}^{2} \delta(z)$, т. е. пропорциональное $Ф Г$ уравнения 
Гельмгольца. Очевидно, решение ИУ (9) удовлетворяет уравнению Гельмгольца $\left(\partial_{z}^{2}+k_{0}^{2}\right) E_{x}(z)=-k_{0}^{2}(\varepsilon(z)-1) E_{x}(z)$ или $\left(\partial_{z}^{2}+k_{0}^{2} \varepsilon(z)\right) E_{x}(z)=0$ как вне, так и внутри пластины. ИУ (9) можно решать методом последовательных приближений, но его хорошая сходимость гарантирована только при близких к единице ДП. Удобно искать решение (9) в виде

$$
E_{x}(z)=E^{+}(z) \exp \left(-i k_{0} \sqrt{\varepsilon(z)} z\right)+E^{-}(z) \exp \left(i k_{0} \sqrt{\varepsilon(z)} z\right),
$$

что сразу позволяет определить $v_{\mathrm{E}}(z)$, но требует численного вычисления амплитуд $E^{ \pm}(z)$. Можно использовать и ВКБ приближение. Для нестационарного туннелирования также можно использовать ИУ, основанные на ФГ. Нестационарная одномерная функция Грина дается обратным Фурье-обращением функции $k_{0}^{-2} G_{\omega}(z)$ и пропорциональна функции Хэвисайда $\chi(t-|z| / c)$. Эта функция и определяет причинность: возникновение сигнала в точке $z^{\prime}$ в момент $t^{\prime}$ не может появится в точке $z$ раньше времени $t=t^{\prime}+\left|z-z^{\prime}\right| / c$. Поляризацию в ИУ следует определять с учетом (1). Однако созданные локализованными источниками поля частот заменяют ВП. Если пакет имеет резкий фронт, проблем с определением времени его прохода какой-либо области не возникает: оно всегда не менее, чем $d / c$ [56]. Но пакет может иметь нерезкий и даже бесконечный передний фронт [27], и тогда возникает проблема введения скорости и времени прохождения им заданной области [57], что является темой отдельного рассмотрения.

Одномерное стационарное уравнение Шредингера можно записать в виде $\left(\partial_{z}^{2}+2 m \mathrm{E}(1-V / \mathrm{E}) / \hbar^{2}\right) \psi(z)=0$. Обозначая $2 m E / \hbar^{2}=k^{2}, 1-V / \mathrm{E}=\varepsilon$, видим, что туннелирование ЭМВ при $\varepsilon<0$ эквивалентно туннелированию частиц при $\mathrm{E}<V$, а потенциальной яме $V<0$ соответствует пластина диэлектрика с $\varepsilon>0$. Такая пластина, как известно, при достаточной толщине представляет собой открытый диэлектрический резонатор, имеющий комплексные частоты. Для матрицы рассеяния возникают полюса в комплексной области частот [58]. Зная $T(\omega)$ и спектр падающего ВП $\Phi(\omega)$, можно вычислить прошедшее поле, взяв обратное преобразование Фурье от $T(\omega) \Phi(\omega)$. В силу расположения полюсов у $T(\omega)$ сверхсветовых откликов не возникает [56]. Для квантовой частицы полюса соответствуют квазистационарным уровням энергии с конечным временем жизни. При рассеянии падающий поток частиц имеет вид $\psi^{+}(z)=A \exp (i k z)$. Величина $\left|\psi^{+}(z)\right|^{2}=|A|^{2}$ дает плотность частиц в потоке. Ищем величины $\psi^{-}(z)=A R \exp (-i k z)$ слева и справа $\psi^{+}(z)=A T \exp (-i k(z-d))$, а также решение $\psi(z)=A^{+} \exp (i k \sqrt{\varepsilon} z)+$ $+A^{-} \exp (-i k \sqrt{\varepsilon} z)$. Очевидно, $|\psi(z)|^{2}$ дает плотность частиц внутри области. Она определяет плотность энергии. Величина $j=(\hbar / m) \operatorname{Im}\left(\psi^{*} \partial_{z} \psi\right)$ определяет поток плотности вероятности, поэтому $v_{E}=j /|\psi|^{2}$. Действительно, в падающем потоке $j^{+}=k \hbar(2 m)^{-1}|A|^{2}$ и величина $v_{\mathrm{E}}=\sqrt{\mathrm{E} /(2 m)}$ определяет скорость частиц потока. Отметим, что в многоскоростных потоках происходит интерференция волн, поэтому энергетическая скорость изменяется. Многоскоростной поток характерен для ВП. Он также возникает при наличии обратного односкоростного потока, например, для волновой функции перед барьером $\psi(z)=A[\exp (i k z)+R \exp (-i k z)]$. Представим коэффициент отражения в показательной форме $R=|R| \exp (i \varphi)$. Для туннелирования обычно $|R| \approx 1$, поэтому $|\psi(z)|^{2}$ имеет минимумы и максимумы, тогда как поток постоянен $-j(z)=v_{\mathrm{E}}|A T|^{2}$. В точках минимума отношение $j(z) /|\psi(z)|^{2}$ может 
стать сверхсветовым, а в точках максимума оно меньше $v_{\mathrm{E}}$. Нетрудно, однако, проверить, что усреднение на длине де-Бройля приведет к постоянному значению скорости $v_{\mathrm{E}}$. Это же справедливо и для монохроматической ЭМВ, которая движется в вакууме. Таким образом, при наличии прямого и отраженного потоков необходимо провести усреднение и в пространстве по длине волны.

Вопрос о времени туннелирования встал после работ Г. А. Гамова (1928), Э. У. Кондона $(1928,1930)$, Ф. Т. Смита (1960), Т. Е. Хартмана (1962), Дж. Р. Флетчера (1985) и последующей лавины работ по туннелированию. Он открыт до сих пор, поскольку имеет место огромное число публикаций по сверхсветовому туннелированию ЭМВ, в том числе и в последнее время, а также и по сверхсветовому квантовому туннелированию. Известен парадокс Хартмана [2,4,23,27-38], заключающийся в насыщении с ростом толщины барьера $d$ времени Бома-Вигнера (времени групповой задержки), получающейся из метода стационарной фазы для коэффициента пропускания T. В публикациях вводятся времена: Бома-Вигнера, или групповое время $\tau_{B W}=\partial_{\omega} \phi$, Буттикера-Ландауэра время $\tau_{B L}=-\hbar \partial_{V} \ln (|T|)$, ларморово время туннелирования $\tau_{L}=-\hbar \partial_{V} \phi$, время Поллака-Миллера $\tau_{P M}=\partial_{\omega} \ln (|T|)[26]$. Вводятся и другие (в том числе комплексные) времена. Величина $V$ соответствует величине потенциального барьера при туннелировании частицы. Применение вышеупомянутых времен к квантовому туннелированию с широким прямоугольным барьером высоты $V$ дает

$$
\begin{gathered}
\tau_{L}=-\hbar \partial_{V} \phi=\frac{\hbar}{V \sqrt{V / \mathrm{E}-1}}, \\
\tau_{B L}=-\hbar \partial_{V} \ln (|T|)=\frac{(V / \mathrm{E}-1)\left(\hbar / V+d \sqrt{\mu_{e} / \mathrm{E}}\right)-\hbar / V}{2(V / \mathrm{E}-1)^{3 / 2}} \\
\tau_{B W}=\tau_{p}=\hbar \partial_{\mathrm{E}} \phi=\hbar /\left(\sqrt{V \mathrm{E}-\mathrm{E}^{2}}\right) \\
\tau_{P M}=\hbar \partial_{\mathrm{E}} \ln (|T|)=\frac{(V / \mathrm{E}-1)\left(\hbar+d \sqrt{\mu_{e} \mathrm{E}}\right)-\hbar}{2 \mathrm{E}(V / \mathrm{E}-1)^{3 / 2}}
\end{gathered}
$$

что приводит к сверхсветовым скоростям [1-29]. Однако такие применения и соответствующие времена никак не отражают кинематику движения частиц в потоке и динамику движения ВП, поскольку изменение фазы коэффициента прохождения связано с интерференцией отраженных волн. В литературе нет предпочтения какому-либо из них. Часто времена (10) с указанными выше заменами и с учетом соотношения $\mathrm{E}=\hbar \omega$ применяют и к туннелированию ЭМВ. Очевидно, для квантового туннелирования электронов через прямоугольный барьер

$$
v_{\mathrm{E}}(z)=2 v /\left[\left(1+\kappa^{2}\right) \cosh (2 \chi(z-d))+\left(1-\kappa^{2}\right)\right]
$$

где $v=\sqrt{\mathrm{E} / \mu_{e}}-$ скорость частиц потока, $\kappa=\sqrt{\mathrm{E} /(V-\mathrm{E})}, \chi=\sqrt{\mu_{e}(V-\mathrm{E}) / \hbar^{2}}$, $\mu_{e}$ - удвоенная масса. Вычисленное для (11) время, согласно (8), для широкого барьера экспоненциально большое. Для него пишем

$$
\tau=\int_{0}^{d} \frac{2 v d z}{\left(1+\kappa^{2}\right) \cosh (2 \chi(z-d))+\left(1-\kappa^{2}\right)}
$$


Интересно отметить, что $v_{\mathrm{E}}(d)=v_{z}$, т. е. электрон как бы проходит барьер, не теряя скорости и энергии. Это же значение $\tau_{\mathrm{E}}=d / v$ имеет место и для очень узкого барьера. За началом широкого барьера для малых $z$ скорость (11) может быть очень малой, поскольку в этой области $|\psi(z)|^{2}$ максимальна внутри барьера. Действительно, амплитуда $A^{-}$экспоненциально мала. Пренебрегая нарастающей волной, имеем

$$
|\psi(z)|^{2}=|T|^{2}\left(1+\kappa^{2}\right) \exp (2 \chi(d-z)) / 4
$$

Именно такая функция при отбрасывании $A^{-}$используется в ВКБ приближении для определения прозрачности барьера $|T|^{2}$.

Таким образом, парадокс Хартмана легко объясним. Сверхсветовых физических движений быть не может [57], хотя фазовые и групповые скорости могут быть сверхсветовыми. Ошибка Хартмана заключается в использовании узкого гауссова пакета волновых функций до барьера, внутри барьера и за барьером, причем раздельно (независимо) для каждой области так, как при решении методом сшивания для стационарного уравнения Шредингера с последующим применением метода стационарной фазы к выходному пакету. Такая ВФ практически соответствует стационарному туннелированию. Но волновая функция нестационарного уравнения Шредингера нелокальная и единая во всех областях. Она удовлетворяет интегральному уравнению, зависящему от потенциала, и изменяется во времени во всем пространстве [59]. Применять метод стационарной фазы в задачах туннелирования и прохождения волн при наличии отражений некорректно. При этом Хартман использовал узкий по спектру гауссов ВП, т.е. фактически задача была сведена к стационарному случаю, где время Бома-Вигнера не соответствует динамике процесса.

В последнее время в экспериментах по сверхсветовому туннелированию фотонов используют интерференцию узких ВП вместо детектирования фотонов. Например, в эксперименте в Беркли [30] использован двухфотонный распад, или параметрическое преобразование с понижением частоты в нелинейном кристалле и образованием двух пакетов. Прошедший среду ВП интерферирует с базовым в НОМ-интерферометре. Однако прошедший слой вещества ВП не является исходным фотоном: он взаимодействовал с веществом с образованием квазифотонов. В этих экспериментах использовался образец в виде многопериодного диэлектрического ФК с двумя слоями в периоде и получено сверхсветовое время. Интересно отметить, что в каждом слое скорость энергии существенно меньше $c$, а результат интерпретируется как распространение света быстрее света. Для определения времени туннелирования частиц в последнее время использованы методы фотоионизации атомов, в частности атомов водорода, методом attoclock [36]. Этот метод использован также в [31-35]. Для них получены весьма малые или даже нулевые времена возникновения частиц с энергиями континуума. Однако фотоионизация приводит к коллапсу волновой функции, что отмечали и авторы эксперимента, и ее трудно сопоставить с обычным туннелированием. Вообще определение времени в квантовом туннелировании связано с определением положения частицы, т. е. с коллапсом волновой функции. Любое такое детектирование связано с введением дополнительного потенциала. 


\section{Заключение}

В стационарном случае времени нет, и некорректно говорить о времени туннелирования отдельной частицей. Для ЭВМ в среде это квазифотоны или поляритоны, соответствующие многофотонным и многочастичным взаимодействиям. Но скорости в потоке определить можно, и в этом смысле поставить их в соответствие частицам потока. В конечной области с изменяющимся потенциалом можно решать задачу, например, методом совместного решения уравнения Шредингера с уравнением Пуассона или методом теории функционала плотности, задавая граничные потоки. В нестационарном случае все величины зависят от времени, включая скорости и времена какихто движений. Введение скоростей для ВП не является однозначным. Задачи нестационарного туннелирования весьма важны для определения переходных процессов в электронных устройствах, но представляют тему отдельного рассмотрения.

Конкурирующие интересы. Конкурирующих интересов не имею.

Авторская ответственность. Я несу полную ответственность за предоставление окончательной версии рукописи в печать. Окончательная версия рукописи мною одобрена.

Финансирование. Работа выполнена при частичной финансовой поддержке гранта Российского научного фонда, проект № 16-19-10033.

\section{Библиографический список}

1. Smith F. T. Lifetime matrix in collision theory // Phys. Rev., 1960. vol.118, no. 6. pp. 349356. doi: 10.1103/PhysRev.119.2098.4.

2. Hartman T. E. Tunneling of a wave packet // J. Appl. Phys., 1962. vol. 33, no. 12. pp. 34273433. doi: 10.1063/1.1702424.

3. Fletcher J. R. Time delay in tunnelling through a potential barrier // J. Phys. C: Solid State Phys., 1985. vol. 18, no. 2. pp. L55-L59. doi: 10.1088/0022-3719/18/2/004.

4. Büttiker M., Landauer R. Traversal time for tunneling// Phys. Rev. Lett., 1982. vol. 49, no. 23. pp. 1739-1742. doi: 10.1103/PhysRevLett.49.1739.

5. Büttiker M. Larmor precession and the traversal time for tunneling// Phys. Rev. B, 1983. vol. 27, no. 10. pp. 6178-6188. doi: 10.1103/PhysRevB.27.6178.

6. Büttiker M., Landauer R. J. Comment on 'The quantum mechanical tunnelling time problem-revisited'// J. Phys. C: Solid State Phys., 1988. vol.21, no.36. pp. 6207-6213. doi : 10.1088/0022-3719/21/36/020.

7. Steinberg A. M., Kwiat P. G., Chiao R. Y. Measurement of the single-photon tunneling time// Phys. Rev. Lett., 1993. vol.71, no.5. pp. 708-711. doi: 10.1103/PhysRevLett.71. 708.

8. Chiao R. Y. Superluminal (but causal) propagation of wavepackets in transparent media with inverted atomic populations // Phys. Rev. A, 1993. vol. 48, no.1. pp. R34-R377. doi: 10.1103/PhysRevA.48.R34.

9. Hass K., Busch P. Causality of superluminal barrier traversal// Phys. Lett. A, 1994. vol. 185, no. 1. pp. 9-13. doi : 10.1016/0375-9601(94)90979-2.

10. Steinberg A. M. Conditional probabilities in quantum theory, and the tunneling time controversy// Phys. Rev. A, 1995. vol.52, no.1. pp. 32-42, arXiv: quant-ph/9502003. doi: 10.1103/PhysRevA.52.32.

11. Chiao R. Y. Tachyonlike excitations in inverted two-level media// Phys. Rev. Lett., 1996. vol. 77, no. 7. pp. 1254-1957. doi: 10.1103/PhysRevLett.77.1254.

12. Olkhovsky V. S., Recami E., Raciti F., Zaichenko A. K. More about tunnelling times, the 
dwell time and the "Hartman effect" // J. Phys. I France, 1995. vol. 5, no. 10. pp. 1351-1365, arXiv: quant-ph/9508010. doi: 10.1051/jp1:1995202.

13. Jakiel J., Olkhovsky V. S., Recami E. On superluminal motions in photon and particle tunnellings // Phys. Lett. A, 1998. vol.248, no. 2-4. pp. 156-162, arXiv: quant-ph/9810053. doi : 10.1016/S0375-9601(98)00626-4.

14. Sassoli de Bianchi M. A simple semiclassical derivation of Hartman's effect // Eur. J. Phys., 2000. vol. 21. pp. L21-L23. doi: 10.1088/0143-0807/21/4/101.

15. Longhi S., Marano M., Laporta P., Belmonte M. Superluminal optical pulse propagation at $1.5 \mathrm{\mu m}$ in periodic fiber Bragg gratings// Phys. Rev. E, 2001. vol.64, no. 5, 055602. doi: 10.1103/PhysRevE.64.055602.

16. Olkhovsky V. S., Recami E., Salesi G. Superluminal tunnelling through two successive barriers // EPL - Europhys. Lett., 2002. vol. 57, no. 6. pp. 879-884, arXiv: quant-ph/0002022. doi: 10.1209/epl/i2002-00592-1.

17. Winful H. Delay time and the Hartman effect in quantum tunneling// Phys. Rev. Lett., 2003. vol. 91, no. 26, 260401. doi: 10.1103/PhysRevLett.91.260401.

18. Olkhovsky V. S., Recami E., Jakiel J. Unified time analysis of photon and particle tunnelling// Phys. Rep., 2004. vol.398, no. 3. pp. 133-178. doi: 10.1016/j.physrep. 2004.06. 001.

19. Martinez J. C., Polatdemir E. Origin of the Hartman effect// Phys. Lett. A, 2006. vol. 351, no. 1-2. pp. 31-36. doi: 10.1016/j.physleta.2005.10.076.

20. Enders A., Nimtz G. Evanescent-mode propagation and quantum tunneling// Phys. Rev. E, 1993. vol. 48, no. 1. pp. 632-634. doi: 10.1103/PhysRevE.48.632.

21. Nimtz G. On superluminal tunneling // Progress in Quantum Electronics, 2003. vol. 27, no. 6. pp. 417-450. doi : 10.1016/S0079-6727(03)00057-0.

22. Nimtz G. Superluminal signal velocity and causality// Found. Phys., 2004. vol. 34, no. 12. pp. 1889-1903. doi : 10.1007/s10701-004-1625-2.

23. Nimtz G. Tunneling confronts special relativity// Found. Phys., 2011. vol.41, no. 7. pp. 1193-1199. doi: 10.1007/s10701-011-9539-2.

24. Hauge E. H., Støvneng J. A. Tunneling times: a critical review // Rev. Mod. Phys., 1989. vol. 61, no. 4. pp. 917-936. doi: 10.1103/RevModPhys.61.917.

25. Winful H. G. Tunneling time, the Hartman effect, and superluminality: A proposed resolution of an old paradox // Phys. Rep., 2006. vol.436, no.1-2. pp. 1-69. doi: 10.1016/j . physrep. 2006.09.002.

26. Yamada N. Unified derivation of tunneling times from decoherence functionals // Phys. Rev. Lett., 2004. vol.93, no. 17, 170401. doi: 10.1103/PhysRevLett.93.170401.

27. Халфин Л. А. Квантовая теория рассеяния волновых пакетов, принцип причинности и сверхсветовое туннелирование // УФН, 1996. Т. 166, №6. С. 688-690. doi: 10.3367/ UFNr.0166.199606j.0688.

28. Шварцбург А. Б. Туннелирование электромагнитных волн - парадоксы и перспективы // УФН, 2007. Т. 177, №1. C. 43-58. doi : 10.3367/UFNr.0177.200701b.0043.

29. Шварцбург А. Б., Ерохин Н. С. Резонансное туннелирование сверхкоротких электромагнитных импульсов в градиентных метаматериалах: парадоксы и перспективы // УФH, 2011. Т. 181, №11. С. 1212-1217. doi: 10.3367/UFNr.0181.201111j.1212.

30. Chiao R. Y., Kwiat P. G., Steinberg A. M. Quantum nonlocality in two-photon experiments at Berkeley// Quantum Semiclass. Opt.. vol. 7, no. 3, arXiv: quant-ph/9501016. doi: 10. 1088/1355-5111/7/3/006.

31. Eckle P., Pfeiffer A. N., Cirelli C., et al. Attosecond ionization and tunneling delay time measurements in Helium // Science, 2009. vol.322, no.5907. pp. 1525-1529. doi: 10.1126/ science.1163439.

32. Pfeiffer A. N., Cirelli C., Smolarski M., et al. Attoclock reveals natural coordinates of the laser-induced tunnelling current flow in atoms // Nat. Phys., 2012. vol. 8. pp. 76-80. doi: 10. $1038 /$ nphys 2125 . 
33. Camus N., Yakaboylu E., Fechner L., et al. Experimental evidence for quantum tunneling time// Phys. Rev. Lett., 2017. vol.119, no. 2, 023201. doi: 10.1103/PhysRevLett.119. 023201.

34. Landsman A. S., Weger M., Maurer J., et al. Ultrafast resolution of tunneling delay time // Optica, 2014. vol. 1, no. 5. pp. 343-349. doi: 10.1364/OPTICA.1.000343.

35. Sainadh U. S., Xu H., Wang X., et al. Attosecond angular streaking and tunnelling time in atomic hydrogen // Nature, 2019. vol.568. pp. 75-77. doi : 10.1038/s41586-019-1028-3.

36. Mitchell M. W., Chiao R. Y. Causality and negative group delays in a simple bandpass amplifier // Amer. J. Phys., 1998. vol.66, no. 1. pp. 14-19. doi: 10.1119/1.18813.

37. Borjemscaia N., Polyakov S. V., Lett P. D., Migdall A. Single-photon propagation through dielectric bandgaps // Optics Express, 2010. vol. 18, no. 3. pp. 2279-2286. doi : 10.1364/OE. 18.002279 .

38. Muga J. G., Palao J. P. Negative time delays in one dimensional absorptive collisions// Annalen der Physik, 1998. vol.7, no.7-8. pp. 671-678. doi:10.1002/(SICI) 1521-3889(199812) 7:7/8<671: :AID-ANDP671>3.0.CO;2-T.

39. Muga J. G., Egusquiza I. L., Damborenea J. A., Delgado F. Bounds and enhancements for negative scattering time delays // Phys. Rev. A, 2002. vol.66, no. 4, 042115. doi: 10.1103/ PhysRevA.66.042115.

40. Dogariu A., Kuzmich A., Wang L. H. Transparent anomalous dispersion and superluminal light-pulse propagation at a negative group velocity// Phys. Rev. A, 2001. vol.63, no. 5, 053806, arXiv: arXiv:physics/0012060 [physics.optics]. doi : 10.1103/PhysRevA.63.053806.

41. Wang L. J., Dogariu A., Kuzmich A. Superluminal light pulse propagation at a negative group velocity / Coherence and Quantum Optics VIII; eds. N. P. Bigelow, J. H. Eberly, C. R. Stroud, I. A. Walmsley. Boston, MA: Springer, 2003. pp. 619-620. doi: 10.1007/ 978-1-4419-8907-9_35.

42. Давидович М. В. Прохождение сигналов через фильтр с поглощением и отрицательное время задержки // ЖТФ, 2012. Т. 82, № 3. С. 15-22.

43. Ландау Л. Д., Лифшиц Е. М. Электродинамика сплошных сред. М.: Наука, 1982.

44. Давидович М. В. О плотности электромагнитной энергии и ее скорости в среде с аномальной положительной дисперсией // Писъма в ЖТТ, 2006. Т. 32, № 22. С. 53-63.

45. Ахиезер А. И., Ахиезер И. А. Электромагнетизм и электромагнитные волны. М.: Высш. шк., 1985. 504 с.

46. Давидович М. В. Законы сохранения и плотности энергии и импульса электромагнитного поля в диспергирующей среде // Изв. Сарат. ун-та. Нов. сер. Сер. Физика, 2012. T. 12, № 1. С. 46-54.

47. Давидович М. В. О плотности электромагнитной энергии и ее скорости в среде с дисперсией, обусловленной проводимостью // ЖТФ, 2010. Т. 80, № 5. С. 40-44.

48. Рытов С. М. Некоторые теоремы о групповой скорости электромагнитных волн // ЖЭТФ, 1947. Т. 176, № 10. С. 930-936.

49. Давидович М. В. Плазмоны в многослойных плоскослоистых структурах // Квантовая электроника, 2017. Т. 47, №6. С. 567-579.

50. Прудников А. П., Брычков Ю. А., Маричев О. И. Интегралы и ряды. Элементарные функции. М.: Наука, 1981. 800 с.

51. Градштейн И. С., Рыжик И. М. Таблицы интегралов, сумм, рядов и произведений. М.: ГИФМЛ, 1962. 1100 с.

52. Давидович М. В. Матрицы рассеяния и передачи неоднородного слоя // Радиотехника и электроника, 2010. Т. 55, № 1. С. 105-112.

53. Давидович М. В. О малоотражающих магнитодиэлектрических покрытиях с экспоненциально зависящими проницаемостями // Радиотехника и электроника, 2010. Т. 55, № 4. С. 492-496.

54. Давидович М. В., Стефюк Ю. В. Нелинейное прохождение электромагнитной волны через слой с квадратичной и дробно-полиномиальной зависимостями диэлектрической 
проницаемости // Известия вузов. ПНД, 2010. Т. 18, № 3. С. 160-177. doi: 10.18500/ 0869-6632-2010-18-3-160-177.

55. Вайнштейн Л. А. Распространение импульсов // УФН, 1976. Т. 118, № 2. С. 339-366. doi: 10.3367/UFNr.0118.197602h.0339.

56. Давидович М. В. О парадоксе Хартмана, туннелировании электромагнитных волн и сверхсветовых скоростях (отклик на статью Шварцбурга А. Б. "Туннелирование электромагнитных волн - парадоксы и перспективы") // УФН, 2009. Т. 179, № 4. С. 443-446. doi: 10.3367/UFNr.0179.200904o.0443.

57. Базь А. И., Зельдович Я. Б., Переломов А. М. Рассеяние, реакиии и распады в нерелятивистской квантовой механике. М.: Наука, 1971. 530 с.

58. Грибов В. Н. Квантовая электродинамика. Москва, Ижевск: Регулярная и хаотическая динамика, 2001. 288 с.

59. Гришаков К. С., Елесин В. Ф. Времена перехода резонансно-туннельного диода между экстремальными точками гистерезисной вольт-амперной характеристики // Физика u техника полупроводников, 2016. Т. 50, № 8. С. 1113-1117. 


\title{
MSC: 34B60, 35Q40, 81Q99
}

\section{The energy transfer velocity by a plane monochromatic electromagnetic wave through a layer of matter}

\author{
M. V. Davidovich \\ N. G. Chernyshevsky Saratov State University (National Research University), \\ 83, Astrakhanskaya st., Saratov, 410012, Russian Federation.
}

\begin{abstract}
Stationary problems for the diffraction (tunneling) of a plane electromagnetic wave through a layer of matter with dielectric properties, as well as a quantum particle tunneling through a rectangular potential barrier are considered. It is shown that there are no superluminal motions, and the transit time is always longer when the wave passes the structure at the speed of light.
\end{abstract}

Keywords: energy velocity, tunneling time, frequency dispersion, BohmWigner time, Hartman paradox.

Received: $22^{\text {nd }}$ August, 2019 / Revised: 19 ${ }^{\text {th }}$ November, $2019 /$

Accepted: $10^{\text {th }}$ February, $2020 /$ First online: $1^{\text {st }}$ April, 2020

Competing interests. I declare that I have no competing interests.

Author's Responsibilities. I take full responsibility for submitting the final manuscript in print. I approved the final version of the manuscript.

Funding. This work was supported in part by a grant from the Russian Science Foundation, project no. 16-19-10033.

\section{References}

1. Smith F. T. Lifetime matrix in collision theory, Phys. Rev., 1960, vol. 118, no. 6, pp. 349-356. doi: 10.1103/PhysRev.119.2098.4.

2. Hartman T. E. Tunneling of a wave packet, J. Appl. Phys., 1962, vol. 33, no. 12, pp. 34273433. doi: 10.1063/1.1702424.

3. Fletcher J. R. Time delay in tunnelling through a potential barrier, J. Phys. C: Solid State Phys., 1985, vol. 18, no. 2, pp. L55-L59. doi: 10.1088/0022-3719/18/2/004.

4. Büttiker M., Landauer R. Traversal time for tunneling, Phys. Rev. Lett., 1982, vol. 49, no. 23, pp. 1739-1742. doi: 10.1103/PhysRevLett.49.1739.

\section{Research Article}

๖ () (i) The content is published under the terms of the Creative Commons Attribution 4.0 International License (http://creativecommons.org/licenses/by/4.0/)

Please cite this article in press as:

Davidovich M. V. The energy transfer velocity by a plane monochromatic electromagnetic wave through a layer of matter, Vestn. Samar. Gos. Tekhn. Univ., Ser. Fiz.-Mat. Nauki [J. Samara State Tech. Univ., Ser. Phys. Math. Sci.], 2020, vol. 24, no. 1, pp. 22-40. doi: 10.14498/vsgtu1741 (In Russian).

\section{Author's Details:}

Mikhail V. Davidovich (1) https://orcid.org/0000-0001-8706-8523

Dr. Phys. \& Math. Sci.; Professor; Dept. of Radio Engineering and Electrodynamics; email: DavidovichMV@info.sgu.ru 
5. Büttiker M. Larmor precession and the traversal time for tunneling, Phys. Rev. B, 1983, vol. 27, no. 10, pp. 6178-6188. doi: 10.1103/PhysRevB.27.6178.

6. Büttiker M., Landauer R. J. Comment on 'The quantum mechanical tunnelling time problem-revisited', J. Phys. C: Solid State Phys., 1988, vol.21, no.36, pp. 6207-6213. doi : 10.1088/0022-3719/21/36/020.

7. Steinberg A. M., Kwiat P. G., Chiao R. Y. Measurement of the single-photon tunneling time, Phys. Rev. Lett., 1993, vol.71, no. 5, pp. 708-711. doi: 10.1103/PhysRevLett.71.708.

8. Chiao R. Y. Superluminal (but causal) propagation of wavepackets in transparent media with inverted atomic populations, Phys. Rev. A, 1993, vol.48, no. 1, pp. R34-R377. doi: 10. 1103/PhysRevA.48.R34.

9. Hass K., Busch P. Causality of superluminal barrier traversal, Phys. Lett. A, 1994, vol. 185, no. 1, pp. 9-13. doi: 10.1016/0375-9601(94)90979-2.

10. Steinberg A. M. Conditional probabilities in quantum theory, and the tunneling time controversy, Phys. Rev. A, 1995, vol. 52, no. 1, pp. 32-42, arXiv: quant-ph/9502003. doi: 10. 1103/PhysRevA.52.32.

11. Chiao R. Y. Tachyonlike excitations in inverted two-level media, Phys. Rev. Lett., 1996, vol. 77, no. 7, pp. 1254-1957. doi: 10.1103/PhysRevLett.77.1254.

12. Olkhovsky V. S., Recami E., Raciti F., Zaichenko A. K. More about tunnelling times, the dwell time and the "Hartman effect", J. Phys. I France, 1995, vol. 5, no. 10, pp. 1351-1365, arXiv: quant-ph/9508010. doi: 10.1051/jp1:1995202.

13. Jakiel J., Olkhovsky V. S., Recami E. On superluminal motions in photon and particle tunnellings, Phys. Lett. A, 1998, vol. 248, no. 2-4, pp. 156-162, arXiv: quant-ph/9810053. doi : 10.1016/S0375-9601(98)00626-4.

14. Sassoli de Bianchi M. A simple semiclassical derivation of Hartman's effect, Eur. J. Phys., 2000, vol. 21, pp. L21-L23. doi: 10.1088/0143-0807/21/4/101.

15. Longhi S., Marano M., Laporta P., Belmonte M. Superluminal optical pulse propagation at $1.5 \mathrm{~mm}$ in periodic fiber Bragg gratings, Phys. Rev. E, 2001, vol. 64, no. 5, 055602. doi: 10 . 1103/PhysRevE.64.055602.

16. Olkhovsky V. S., Recami E., Salesi G. Superluminal tunnelling through two successive barriers, EPL - Europhys. Lett., 2002, vol. 57, no. 6, pp. 879-884, arXiv: quant-ph/0002022. doi: 10.1209/epl/i2002-00592-1.

17. Winful H. Delay time and the Hartman effect in quantum tunneling, Phys. Rev. Lett., 2003, vol. 91, no. 26, 260401. doi: 10.1103/PhysRevLett.91.260401.

18. Olkhovsky V. S., Recami E., Jakiel J. Unified time analysis of photon and particle tunnelling, Phys. Rep., 2004, vol.398, no.3, pp. 133-178. doi: 10.1016/j.physrep.2004.06.001.

19. Martinez J. C., Polatdemir E. Origin of the Hartman effect, Phys. Lett. A, 2006, vol. 351, no. 1-2, pp. 31-36. doi: 10.1016/j.physleta.2005.10.076.

20. Enders A., Nimtz G. Evanescent-mode propagation and quantum tunneling, Phys. Rev. E, 1993, vol. 48, no. 1, pp. 632-634. doi: 10.1103/PhysRevE.48.632.

21. Nimtz G. On superluminal tunneling, Progress in Quantum Electronics, 2003, vol. 27, no. 6, pp. 417-450. doi : 10.1016/S0079-6727(03)00057-0.

22. Nimtz G. Superluminal signal velocity and causality, Found. Phys., 2004, vol. 34, no. 12, pp. 1889-1903. doi : 10.1007/s10701-004-1625-2.

23. Nimtz G. Tunneling confronts special relativity, Found. Phys., 2011, vol. 41, no. 7, pp. 11931199. doi: $10.1007 / \mathrm{s} 10701-011-9539-2$.

24. Hauge E. H., Støvneng J. A. Tunneling times: a critical review, Rev. Mod. Phys., 1989, vol. 61, no. 4, pp. 917-936. doi: 10.1103/RevModPhys.61.917.

25. Winful H. G. Tunneling time, the Hartman effect, and superluminality: A proposed resolution of an old paradox, Phys. Rep., 2006, vol.436, no. 1-2, pp. 1-69. doi: 10.1016/j . physrep. 2006.09.002.

26. Yamada N. Unified derivation of tunneling times from decoherence functionals, Phys. Rev. Lett., 2004, vol.93, no. 17, 170401. doi: 10.1103/PhysRevLett.93.170401. 
27. Khalfin L. A. The quantum theory of wave packet scattering, the causality principle, and superlight tunnelling, Phys. Usp., 1996, vol.39, no.6, pp. 639-642. doi: 10.1070/ PU1996v039n06ABEH001518.

28. Shvartsburg A. B. Tunneling of electromagnetic waves: paradoxes and prospects, Phys. Usp., 2007, vol. 50, no. 1, pp. 37-51. doi : 10.1070/PU2007v050n01ABEH006148.

29. Shvartsburg A. B., Erokhin N. S. Resonant tunneling of ultrashort electromagnetic pulses in gradient metamaterials: paradoxes and prospects, Phys. Usp., 2011, vol. 54, no. 11, pp. 11711176. doi : 10.3367/UFNe.0181.201111j.1212.

30. Chiao R. Y., Kwiat P. G., Steinberg A. M. Quantum nonlocality in two-photon experiments at Berkeley, Quantum Semiclass. Opt., vol. 7, no.3, arXiv: quant-ph/9501016. doi : $10.1088 / 1355-5111 / 7 / 3 / 006$.

31. Eckle P., Pfeiffer A. N., Cirelli C., et al. Attosecond ionization and tunneling delay time measurements in Helium, Science, 2009, vol.322, no. 5907, pp. 1525-1529. doi: 10.1126/ science.1163439.

32. Pfeiffer A. N., Cirelli C., Smolarski M., et al. Attoclock reveals natural coordinates of the laser-induced tunnelling current flow in atoms, Nat. Phys., 2012, vol. 8, pp. 76-80. doi: 10. 1038/nphys2125.

33. Camus N., Yakaboylu E., Fechner L., et al. Experimental evidence for quantum tunneling time, Phys. Rev. Lett., 2017, vol.119, no. 2, 023201. doi: 10.1103/PhysRevLett.119. 023201.

34. Landsman A. S., Weger M., Maurer J., et al. Ultrafast resolution of tunneling delay time, Optica, 2014, vol. 1, no. 5, pp. 343-349. doi: 10.1364/OPTICA.1.000343.

35. Sainadh U. S., Xu H., Wang X., et al. Attosecond angular streaking and tunnelling time in atomic hydrogen, Nature, 2019, vol.568, pp. 75-77. doi: 10.1038/s41586-019-1028-3.

36. Mitchell M. W., Chiao R. Y. Causality and negative group delays in a simple bandpass amplifier, Amer. J. Phys., 1998, vol.66, no. 1, pp. 14-19. doi: 10.1119/1.18813.

37. Borjemscaia N., Polyakov S. V., Lett P. D., Migdall A. Single-photon propagation through dielectric bandgaps, Optics Express, 2010, vol.18, no. 3, pp. 2279-2286. doi: 10.1364/OE. 18.002279 .

38. Muga J. G., Palao J. P. Negative time delays in one dimensional absorptive collisions, Annalen der Physik, 1998, vol.7, no.7-8, pp. 671-678. doi:10.1002/(SICI) 1521-3889 (199812) $7: 7 / 8<671:$ : AID-ANDP671>3.0.C0;2-T.

39. Muga J. G., Egusquiza I. L., Damborenea J. A., Delgado F. Bounds and enhancements for negative scattering time delays, Phys. Rev. A, 2002, vol.66, no.4, 042115. doi: 10.1103/ PhysRevA.66.042115.

40. Dogariu A., Kuzmich A., Wang L. H. Transparent anomalous dispersion and superluminal light-pulse propagation at a negative group velocity, Phys. Rev. A, 2001, vol. 63, no. 5, 053806, arXiv: arXiv:physics/0012060 [physics.optics]. doi : 10.1103/PhysRevA.63.053806.

41. Wang L. J., Dogariu A., Kuzmich A. Superluminal light pulse propagation at a negative group velocity, In: Coherence and Quantum Optics VIII; eds. N. P. Bigelow, J. H. Eberly, C. R. Stroud, I. A. Walmsley. Boston, MA, Springer, 2003, pp. 619-620. doi: 10.1007/ 978-1-4419-8907-9_35.

42. Davidovich M. V. Propagation of signals through a dissipative filter and the negative time delay, Tech. Phys., 2012, vol.57, no. 3, pp. 328-335. doi: 10.1134/S1063784212030048.

43. Landau L. D., Lifshits E. M. Electrodynamics of Continuous Media, Course of Theoretical Physics, vol. 8. New York, Pergamon, 1984.

44. Davidovich M. V. Electromagnetic energy density and velocity in a medium with anomalous positive dispersion, Tech. Phys. Lett., 2006, vol.32, no. 22, pp. 982-986. doi: 10.1134/ S106378500611023X.

45. Akhiezer A. I., Akhiezer I. A. Elektromagnetizm i elektromagnitnye volny [Electromagnetism and Electromagnetic Waves]. Moscow, Vyssh. shk., 1985, 504 pp. (In Russian)

46. Davidovich M. V. The conservation laws and the densities of electromagnetic field energy and momentum in dispersive media, Izv. Saratov Univ. (N. S.), Ser. Physics, 2012, vol. 12, no. 1, pp. 46-54 (In Russian). 
47. Davidovich M. V. On the electromagnetic energy density and energy transfer rate in a medium with dispersion due to conduction, Tech. Phys., 2010, vol.55, no. 5, pp. 630-635. doi : 10.1134/S1063784210050063.

48. Rytov S. M. Some theorems of the group velocity of electromagnetic waves, JETP, 1947, vol. 176, no. 10, pp. 930-936 (In Russian).

49. Davidovich M. V. Plasmons in multilayered plane-stratified structures, Quantum Electronics, 2017, vol.47, no. 6, pp. 567-579. doi: 10.1070/QEL16272.

50. Prudnikov A. P., Brychkov Yu. A., Marichev O. I. Integrals and Series. London, Routledge, 1992. doi : 10.1201/9780203750643.

51. Gradshteyn I. S., Ryzhik I. M. Table of Integrals, Series, and Products. California, Academic Press, 1980. doi : 10.1016/c2013-0-10754-4.

52. Davidovich M. V. Scattering and transmission matrices of an inhomogeneous layer, J. Commun. Technol. Electron., 2010, vol. 55, no. 1, pp. 20-27. doi: 10.1134/S1064226910010031.

53. Davidovich M. V. On low-reflection magnetodielectric coatings with exponential dependences of permittivity and permeability, J. Commun. Technol. Electron., 2010, vol. 55, no. 4, pp. 465-468. doi : 10.1134/S1064226910040133.

54. Davidovich M. V., Stefjuk J. V. Nonlinear electromagnetic wave passing through the layer with quadratic and fractionallypolynomial permittivity dependences on amplitude, Izvestiya VUZ. Applied Nonlinear Dynamics, 2010, vol. 18, no. 3, pp. 160-177 (In Russian). doi : 10. 18500/0869-6632-2010-18-3-160-177.

55. Vainshtein L. A. Propagation of pulses, Sov. Phys. Usp., 1976, vol. 19, no. 2, pp. 189-205. doi : 10.1070/PU1976v019n02ABEH005138.

56. Davidovich M. V. On the Hartman paradox, electromagnetic wave tunneling and supraluminal velocities (comment on the paper "Tunneling of electromagnetic waves: paradoxes and prospects" by A. B. Shvartsburg), Phys. Usp., 2009, vol. 52, no.4, pp. 415-418. doi : 10.3367/UFNe.0179.2009040.0443.

57. Baz' A. I., Zel'dovich Ia. B., Perelomov A. M. Rasseianie, reaktsii i raspady v nereliativistskoi kvantovoi mekhanike [Scattering, Reactions and Decay in Nonrelativistic Quantum Mechanics]. Moscow, Nauka, 1971, 530 pp. (In Russian)

58. Gribov V. N. Kvantovaia elektrodinamika [Quantum Electrodynamics]. Moscow, Izhevsk, Regular and Chaotic Dynamics, 2001, 288 pp. (In Russian)

59. Grishakov K. S., Elesin V. F. Transition times between the extremum points of the current-voltage characteristic of a resonant tunneling diode with hysteresis, Semiconductors, 2016, vol.50, no. 8, pp. 1092-1096. doi: 10.1134/S1063782616080121. 